\title{
Agregación de sedimentos en una cuenca de llanura: utilización del Microscopio Electrónico de Barrido como herramienta de evaluación
}

\section{Aggregation of sediment in a basin plain: the use of Scanning Electron Microscope images as an evaluation tool}

\author{
P. Suarez, O. Orfeo \\ Centro de Ecología Aplicada del Litoral - CONICET, Ruta Provincial N5, Km 2,5 (CP 3400), Corrientes, Argentina. \\ Email: paolasuarez792@gmail.com
}

\section{RESUMEN}

\begin{abstract}
El estudio de materiales movilizados a través de los sistemas fluviales es de gran interés para resolver cuestiones tanto ambientales como de ingeniería. La presencia de sedimentos formando agregados, es frecuente en sistemas fluviales con lechos ricos en materiales finos. La abundante presencia de materia orgánica, la alta concentración de sales, y la baja velocidad de la corriente son algunos factores que favorecen esta agregación, la cual afecta directamente las posibilidades de movilización de los sedimentos. En este trabajo se evaluó la presencia de agregados naturales en los sedimentos de fondo del río Negro, tributario del río Paraná, ubicado en la provincia del Chaco (Argentina). Para ello se realizaron análisis granulométricos en etapas consecutivas de procesamiento de muestras que evaluaron los efectos de la presencia de materia orgánica y las sales disueltas en el estado de agregación de las partículas sedimentarias. En cada etapa las muestras fueron observadas bajo Microscopio Electrónico de Barrido (MEB). Los resultados conseguidos representan la primera evaluación cuantitativa sobre la presencia de agregados naturales de sedimentos en un río de la llanura Chaqueña, pudiéndose usar además como referencia para la caracterización de otros ríos similares. Los resultados obtenidos permiten apreciar un aumento marcado de la fracción limo al eliminar materia orgánica, y de la fracción arcilla al eliminar el efecto electroquímico producto de las altas concentraciones salinas. Se concluye que para estudios destinados a interpretaciones ambientales no es recomendable el uso de técnicas que incluyan eliminación de materia orgánica particulada fina y la dispersión química, pues la información generada puede conducir a una interpretación sesgada de la dinámica de transporte de los sedimentos en el medio natural.
\end{abstract}

Palabras clave: Sedimentos; Formación de agregados; Ríos subtropicales; Granulometría; Imágenes MEB.

\section{ABSTRACT}

The purpose of this study was to determine the presence of sedimentary aggregates, which affect the transport of fine sediments. This contribution explores the influence of organic matter and dissolved salts, as the main factors that determine the aggregation of particles. Bottom sediments of Negro river (tributary of the Paraná River), flows in the Chaco province (North-East of Argentina), and were assessed by granulometric analysis in consecutive phases of sample processing, to evaluate the presence of aggregates. The relative abundance of the different

Recibido el 19 de febrero de 2015 / Aceptado el 10 de agosto de 2015 / Publicado online el 06 de noviembre de 2015

Citation / Cómo citar este artículo: P. Suarez \& O. Orfeo (2015). Agregación de sedimentos en una cuenca de llanura: utilización del Microscopio Electrónico de Barrido como herramienta de evaluación. Estudios Geológicos 71(2): e038. http://dx.doi.org/10.3989/ egeol.42054.366.

Copyright: () 2015 CSIC. This is an open-access article distributed under the terms of the Creative Commons Attribution-Non Commercial (by-nc) Spain 3.0 License. 
sizes in each analyzed phase, was controlled by Scanning Electron Microscope (SEM). The obtained results represent the first quantitative set of values of sediment aggregation in a river of the Chaco Plain, and can be used as a reference for the characterization of other rivers of subtropical plains. The results of this study show that the aggregates formation was favored by the high concentration of dissolved salts, but also by the presence of organic matter, abundant in subtropical environments. It was concluded that it is not convenient to apply classical techniques of sediment particle disaggregation (chemical oxidation to remove organic matter and chemical dispersants to break down associated particles), in order to avoid misinterpretation of transport dynamics of sediments in the natural environment.

Keywords: Sediment aggregation; Subtropical rivers; Granulometric analysis; SEM images.

\section{Introducción}

Los fenómenos de transporte de sedimentos son de especial interés en la hidrología fluvial para resolver tanto cuestiones ambientales como de ingeniería (McLaren \& Bowles, 1984; Habibi, 1994; Ackermann \& Schubert, 2007; Friese et al., 2007; Symader et al., 2007; Kurt et al., 2007; Chetelat et al., 2013; Hostache et al., 2014, entre otros).

Los materiales finos que se movilizan en suspensión a través de los sistemas fluviales deben ser estudiados detenidamente para conocer su procedencia y destino, principalmente en aquellos ambientes que presentan evidencias de contaminación o algún riesgo para el funcionamiento de los ecosistemas.

Los sedimentos, de acuerdo a su facilidad de agregación, pueden ser clasificados en cohesivos y no cohesivos. Aunque no existe una delimitación clara entre ellos, se considera no cohesivos a los sedimentos con tamaño de grano $>60 \mu \mathrm{m}$, mientras que aque$1 \mathrm{los}<2 \mu \mathrm{m}$ (arcillas) son generalmente cohesivos. Si bien los granos de tamaño más fino son considerados los más adherentes, los limos $(2 \mu \mathrm{m}-60 \mu \mathrm{m})$ presentan propiedades aglutinantes debido a la presencia de las arcillas, por lo que en la práctica, limos y arcillas son considerados materiales cohesivos (Huang et al., 2006).

Las partículas finas que forman parte de los materiales cohesivos presentan uniones fuertes entre ellas debido a sus cargas iónicas superficiales, por lo que en estas circunstancias, son las fuerzas entre las partículas, y no la fuerza gravitacional, las que dominan el comportamiento de los sedimentos (Hayter, 1983; Berlamont et al., 1993; Huang et al., 2006).

Es así que los sedimentos de menor tamaño tienden a unirse formando unidades de mayor tamaño, con diferentes posibilidades de transporte y velocidad de sedimentación que las partículas individuales. Este proceso depende del tipo y la concentración de sedimento, de la concentración iónica en la columna de agua (principalmente la salinidad) y de las condiciones del flujo (Mehta et al., 1989; Berlamont et al., 1993; Huang et al., 2006). El contenido de algunos metales (hierro, aluminio y calcio) y de materia orgánica (MO) también influye en los procesos de atracción entre los sedimentos. Estos agregados tienen la particularidad de aumentar de tamaño cuando colisionan con otras partículas o agregados, pero también pueden romperse por efecto de la turbulencia.

Por norma general, las partículas más redondeadas, de menor tamaño y menor densidad son transportadas con mayor facilidad (Spalletti, 1986). El mecanismo principal por el cual los materiales finos se desplazan en los ríos de llanura es el transporte en suspensión, el cual depende de la intensidad del flujo, de su densidad y viscosidad, así como de forma y densidad de los sedimentos. Al agregarse los sedimentos finos, se acelera la velocidad de sedimentación, disminuyendo el tiempo de permanencia de los mismos en la columna de agua, acortando las distancias de transporte y favoreciendo la colmatación de las cuencas. Especial atención merece esta situación, dado que nutrientes y contaminantes se encuentran asociados a sedimentos particulados, por lo que el estudio de dinámica sedimentaria permite además establecer zonas de acumulación y resuspensión de estos materiales asociados. Lo dicho también fue señalado en sedimentos de origen eólico depositados en otros ambientes del noreste argentino (Iriondo et al., 1998; Orfeo, 1998).

En cuanto a los procesos de sedimentación, Berlamont et al. (1993) señalan que en los sedimentos donde las partículas finas se agregan, el tamaño, la densidad y la forma de los flóculos, serán las propiedades que definan la velocidad de sedimentación. Sin embargo, algunas de estas propiedades pueden variar espacial y/o estacionalmente como consecuencia de los diferentes niveles de turbulencia, agregación o desagregación de flóculos, 
la concentración de sedimentos, la abundancia de compuestos orgánicos en el sistema y la química del ambiente (como por ejemplo la concentración iónica).

El análisis clásico que evalúa el transporte de sedimentos en medio líquido suele basarse en la abundancia de partículas individuales por unidad de volumen, para lo cual se elimina previamente la materia orgánica y otros aglutinantes químicos. Sin embargo, los materiales finos en estado natural se encuentran frecuentemente formando agregados que se comportan como granos de mayor tamaño, lo cual requiere mayor capacidad del agente de transporte para su movilización. Es por ello que si los estudios que analizan el transporte sedimentario en ríos tropicales de llanuras no tienen en cuenta este factor, pueden introducir interpretaciones subjetivas sobre la dinámica de los materiales detríticos.

En el presente trabajo se evaluó el efecto de la MO y de las sales en solución, sobre la agregación entre partículas de sedimentos de un río de llanura, donde la vegetación es abundante y el tiempo de permanencia del agua suele ser prolongado, acentuando los procesos de concentración iónica y de sedimentación. Estudios anteriores en el área de estudio señalaron la incidencia salina en la concentración de sedimentos suspendidos, atribuyendo las desviaciones del comportamiento esperado a la probable incidencia de la formación de agregados naturales de partículas de tamaño arcilla (Orfeo, 1999). Los resultados obtenidos en ésta contribución constituyen la primera evaluación cuantitativa realizada sobre la presencia de agregados de sedimentos en ríos de la llanura Chaqueña, y pueden ser usados como referencia para la caracterización de otros ríos de planicie subtropical.

La relación existente entre los fenómenos de adsorción de nutrientes y contaminantes por parte de las partículas finas de los suelos y su relación con las actividades antrópicas que allí se realizan, justifican una especial atención a los estudios de exportación de sedimentos en cuencas con intervención humana.

\section{Área de estudio}

Se seleccionó un tramo del río Negro, localizado en la provincia del Chaco (República Argentina), perteneciente a la cuenca del Paraná. Es un ejemplo característico de áreas con escasa pendiente, con diseño típicamente meandroso, incluido dentro de los Sistemas Hidrológicos No Típicos -SHNT(Fertonani \& Prendes, 1983). Su origen es autóctono, ya que se alimenta de las lluvias locales, siendo el río Paraná su colector final.

Los cuatro puntos de muestreo elegidos (Fig. 1, Tabla 1), representan los diferentes escenarios fluviales: (P1) área de cabecera, con bajo caudal y abundante vegetación de plantas acuáticas y palustres que en ocasiones llegan a cubrir todo el ancho del río. (P2) y (P3) tramo medio, con cauce bien definido, mayor caudal que en la zona de cabecera y abundante vegetación durante la mayor parte del año. (P4) tramo distal cercano a su desembocadura, ubicado aguas abajo de la ciudad de Resistencia, con más del doble de caudal que el registrado en los puntos anteriores, alto impacto antrópico por vertido de efluentes domésticos e industriales (industria frigorífica y láctea) (Ruberto, 1999; Poi et al., 2003), y donde la abundancia de la población vegetal varía a lo largo del año, limitada por lo general a las márgenes del curso.

El área estudiada del río Negro, se encuentra incluida dentro de las $508.000 \mathrm{Ha}$ del Este chaqueño que fueran designadas en 2004 como sitio RAMSAR con el $n^{\circ} 1366$ (RAMSAR).

En el área de estudio, las aguas subterráneas salobres y el contenido salino propio de los suelos, junto con el déficit hídrico que ocasionalmente soporta la región, han generado la presencia de suelos salinos y salino-alcalinos (OEA, 1975; Ledesma \& Zurita, 1995). En la Figura 1 se presenta la distribución de los suelos en el área de muestreo y las zonas cercanas, donde se observa que el tramo muestreado del río Negro atraviesa suelos del orden molisol. Smith (1986) describe los suelos de dicho orden como ricos en materia orgánica y en iones alcalinos con dominancia $\mathrm{Ca}^{++} \mathrm{y} \mathrm{Mg}^{++}$, siendo frecuente encontrar horizontes ricos en acumulación de arcilla que pueden estar formando agregados, así como horizontes ricos en carbonatos cálcico-magnésicos (15\% o más) y horizontes sódicos (con más del 15\% de $\mathrm{Na}^{+}$intercambiable).

\section{Métodos}

Durante un período de dos años se tomaron muestras en los cuatro puntos de muestreo seleccionados, con una periodicidad de seis meses y teniendo en cuenta el ciclo hidrológico: aguas altas (campañas A y C) 


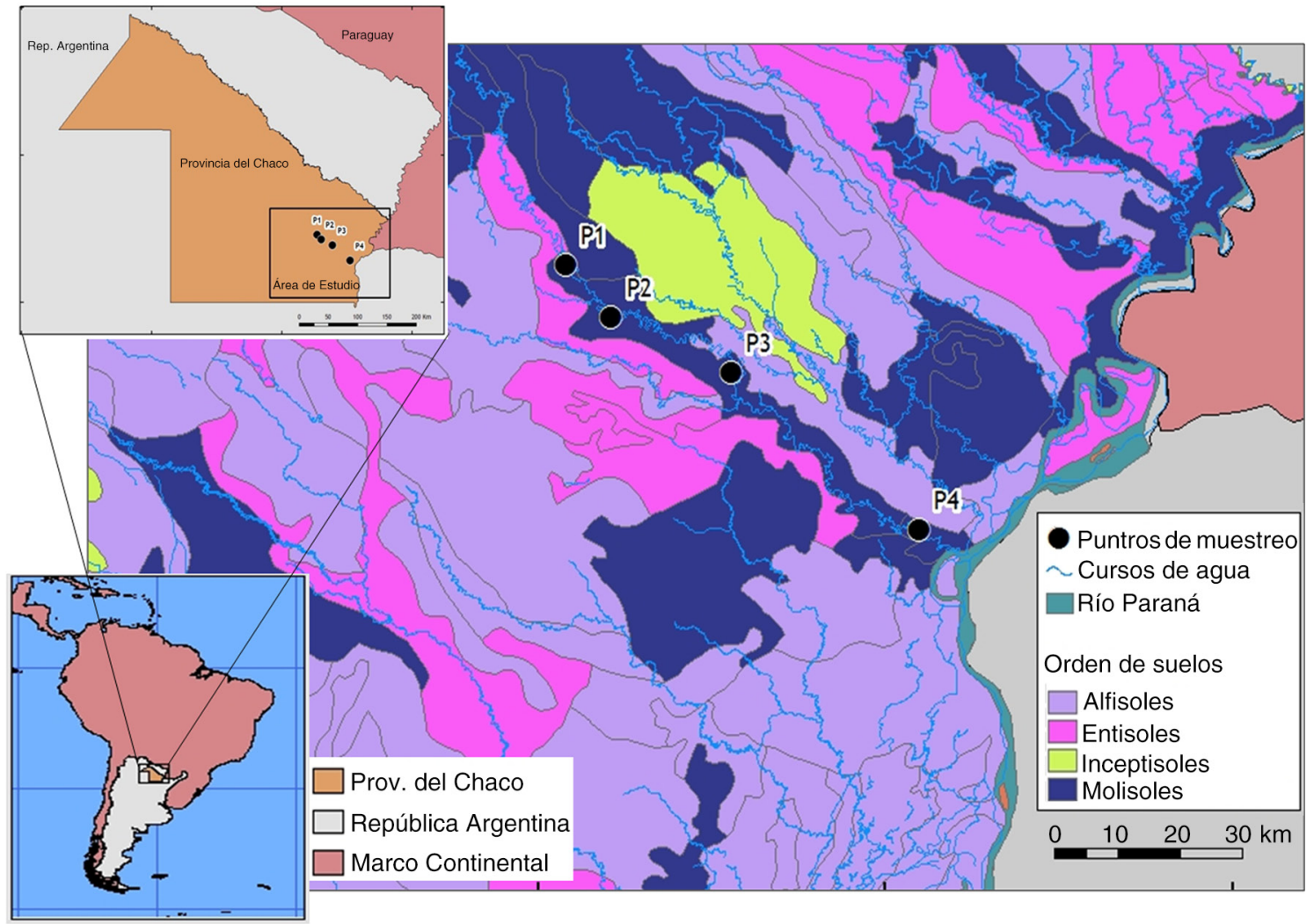

Fig. 1.-Área de estudio.

y aguas bajas (campañas B y D). En cada campaña se extrajeron sedimentos depositados en el centro y ambas márgenes del cauce, utilizando barreno manual.

Adicionalmente se tomaron muestras de agua, utilizando botella de Van Dorn de 1 Litro de capacidad, a las cuales se le midió la conductividad eléctrica y la concentración de sólidos en suspensión.

Para obtener información específica referente a los efectos aglutinantes de la materia orgánica y de la concentración de sales sobre la abundancia relativa de cada clase granulométrica, las muestras fueron tratadas de 3 formas diferentes antes de la cuantificación granulométrica: (T1) sin tratamientos previos:

\section{Tabla 1.-Coordenadas geográficas de los puntos de muestreo}

\begin{tabular}{|c|c|}
\hline Punto de Muestreo & Coordenadas geográficas \\
\hline P1 - Localidad "Colonia Elisa" & $27^{\circ} 04^{\prime} 32^{\prime \prime S} 59^{\circ} 27^{\prime} 23^{\prime \prime} \mathrm{W}$ \\
\hline P2 - Localidad "La Verde" & $27^{\circ} 08^{\prime} 40,5^{\prime \prime S} 59^{\circ} 23^{\prime} 38,8^{\prime \prime} \mathrm{W}$ \\
\hline P3 - Localidad "Laguna Blanca" & $27^{\circ} 13^{\prime} 6,4^{\prime \prime S} 59^{\circ} 13^{\prime} 15,1 " \mathrm{~W}$ \\
\hline P4 - Localidad "Resistencia" & $27^{\circ} 25^{\prime} 49^{\prime \prime} \mathrm{S} 58^{\circ} 56^{\prime} 57,7^{\prime \prime} \mathrm{W}$ \\
\hline
\end{tabular}

cuya finalidad fue conocer el agrupamiento de las partículas sedimentarias tal como se presentan en el medio natural; (T2) eliminación de materia orgánica: mediante la utilización de oxidante químico $\left(\mathrm{H}_{2} \mathrm{O}_{2} 30 \%\right)$ en frío y en caliente (Lafleur 1980), con el propósito de eliminar los agregados sedimentarios causados por la presencia aglutinante de materia orgánica; (T3) eliminación de materia orgánica y dispersión de arcillas: además del tratamiento realizado en la etapa 2 , se trataron las muestras con dispersante químico (hexametafosfato de sodio 4\%) para eliminar el efecto aglutinante residual causado por la concentración salina y que afecta a la fracción arcilla (Lafleur, 1980).

Para conocer la abundancia granulométrica en cada una de las etapas mencionadas anteriormente, se utilizaron análisis granulométricos clásicos (Carver, 1971; McManus, 1995). En cada etapa las muestras fueron observadas en Microscopio Electrónico de Barrido (MEB), con la finalidad de verificar en forma directa la eventual presencia de agregados sedimentarios. 


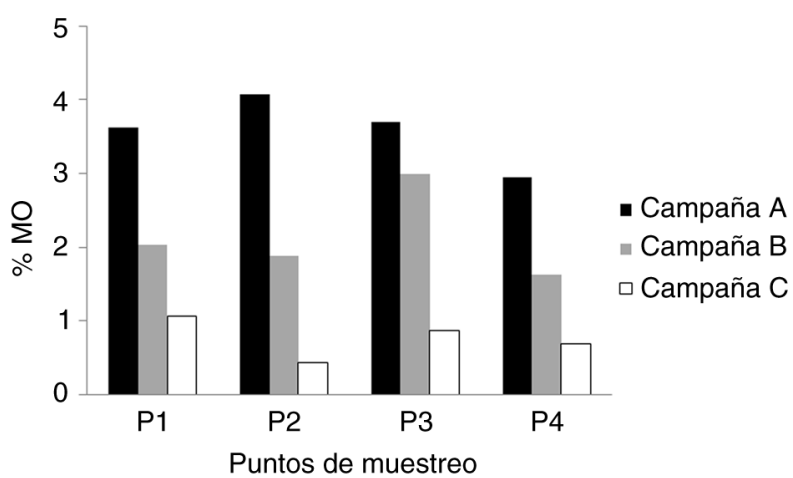

Fig. 2.-Porcentaje de MO en sedimentos del fondo del cauce, en cada punto de muestreo.

\section{Resultados y discusión}

En la Figura 2, se ha representado el porcentaje de materia orgánica en los sedimentos depositados en el fondo del cauce, correspondientes a tres campañas de muestreo. Se han observado valores semejantes a los mencionados por otros autores para humedales de la región y de Iberoamérica (Clemente \& Arrocena, 2003; Davide et al., 2003; Neiff \& Orfeo, 2003). La variación en los porcentajes de materia orgánica observada en este trabajo se encontraría regulada por la estacionalidad climática, con disminución del contenido de $\mathrm{MO}$ al aumentar la oferta pluvial. Este comportamiento probablemente se encuentra relacionado con fenómenos de transporte, que no permiten la acumulación de la materia orgánica en los sedimentos de fondo; lo cual coincide con lo informado por Poi et al. (2003), quienes señalan aumento de la MO durante los períodos de aguas bajas. De acuerdo a lo observado en campo, esta variación se encuentra relacionada con la vegetación palustre que habita estos humedales, la cual aumenta su densidad durante los períodos de estiaje, colonizando gran parte del cuerpo de agua.

La Figura 3 muestra la variación de la conductividad eléctrica del agua (CEA) y su relación con la concentración de sólidos suspendidos (CSS). En dicha figura se indican con color gris los muestreos realizados durante períodos de estiajes y con color blanco los correspondientes a períodos lluviosos. Se observa una variación inversa entre ambas variables, regulada por la estacionalidad climática, lo cual también ha sido observado por otros autores para otros ríos de la zona (Patiño \& Orfeo, 1997; Suárez et al., 2010). Según Orfeo (1999), durante los períodos de aguas bajas es frecuente que la CSS de los ríos aumente; sin embargo, destaca que en algunos cursos (especialmente los de composición hidroquímica predominantemente salina), esta tendencia se invierte. Durante los períodos de estiaje la concentración de sales aumenta, lo cual favorece la aglutinación de materiales finos y acelera los procesos depositacionales. Sin embargo, durante los períodos lluviosos el elevado caudal diluye las sales, y disminuye la CSS.

La Figura 4 muestra la abundancia granulométrica correspondiente a 48 muestras de sedimentos de fondo, recolectadas en los cuatro puntos de muestreo en ambas márgenes y en el centro del cauce. T1 representa las muestras sin tratamiento previo, ello implica que los resultados obtenidos representan la distribución de clases granulométricas de los sedimentos en el ambiente natural. Se aprecia un dominio

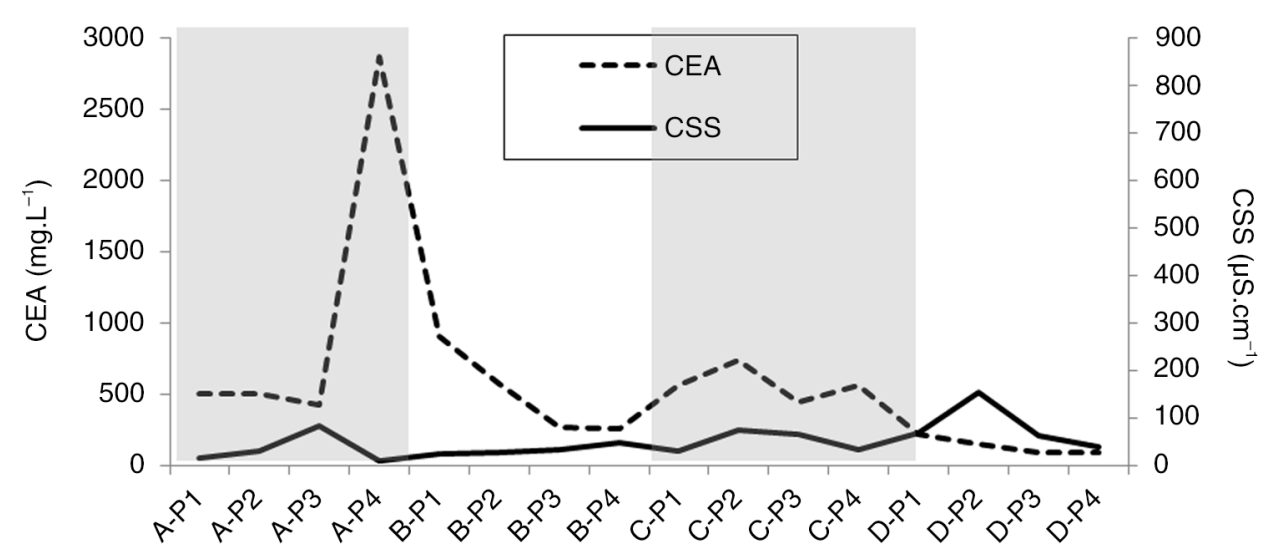

Fig. 3.-Variación longitudinal de la CEA y la CSS. Ref. eje x: letra indica campaña, número indica punto de muestreo. En color gris las campañas durante aguas bajas y en blanco durante aguas altas. 

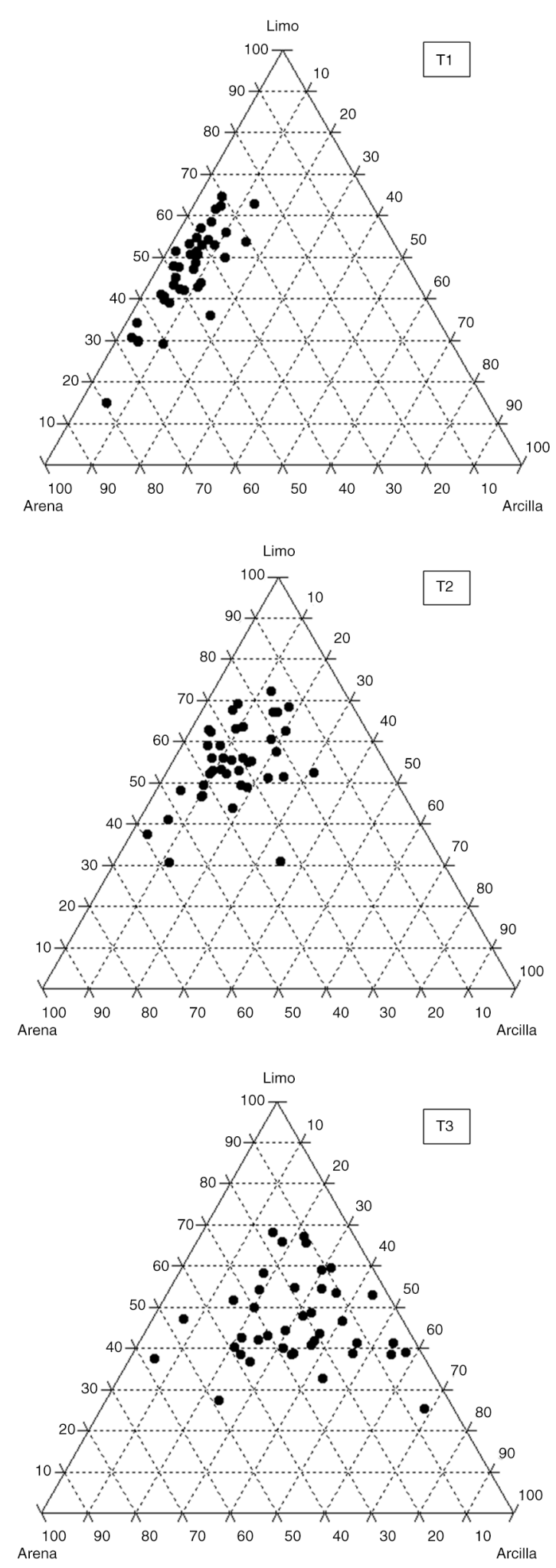

Fig. 4.-Abundancia granulomética (\% en peso) de muestras sin tratamiento previo (T1), de muestras sin $\mathrm{MO}$ (T2) y de muestras sin $\mathrm{MO}$ y con dispersante (T3). de las fracciones de tamaño arena fina $(62-125 \mu \mathrm{m})$ a limo $(62-4 \mu \mathrm{m})$, mientras que la fracción arcilla $(<4 \mu \mathrm{m})$ se encuentra pobremente representada.

Cuando se utiliza oxidante químico para eliminar la agregación del sedimento ocasionada por la materia orgánica (Fig. 4: T2), aumenta principalmente la proporción de arcilla y en menor medida de limo.

La Figura 4: T3 muestra la abundancia granulométrica de los sedimentos de fondo de muestras sin materia orgánica y tratadas con dispersante químico. Se observa un aumento claro de los porcentajes de arcilla en relación al resto de las fracciones.

Del análisis de la Figura 4 (T1, T2 y T3), se desprende que la abundancia de las fracciones con tamaño de partículas superior a $62 \mu \mathrm{m}$ no responde solamente a la presencia de granos de arena, sino también a la existencia de agregados de materiales sedimentarios finos.

La Figura 5 recopila microfotografías tomadas en microscopio electrónico de barrido de los sedimentos de fondo en las tres etapas de análisis granulométrico.

Las imágenes seleccionadas poseen una magnificación de 1500 aumentos, con la cual se favorece la adecuada visualización de todos los tamaños de grano que se encuentran presentes en las diferentes muestras.

La imagen A pertenece a la microscopía de muestras sin tratamiento previo. Se observa una densa agregación de materiales, lo cual le otorga a los agregados de partículas un aspecto rugoso. La microfotografía $\mathrm{B}$ representa la distribución granulométrica luego de eliminar $\mathrm{MO}$, se aprecia una disminución de las rugosidades superficiales, indicando una disminución de agregados. En la imagen $\mathrm{C}$, correspondientes a muestras sin $\mathrm{MO}$ y con dispersantes químico, las partículas individuales se observan en forma desagregada.

Lo anterior permite comprobar que la eliminación de materia orgánica no permite alcanzar resultados totalmente satisfactorios en la determinación de ensayos granulométricos, ya que resulta necesario eliminar también otros factores aglutinantes. Esta observación se corresponde con la Figura 3, que relaciona la conductividad eléctrica del agente de transporte con la concentración de los sólidos suspendidos. Consideraciones semejantes han presentado trabajos anteriores de Gibbs (1983) y Orfeo (1998). 

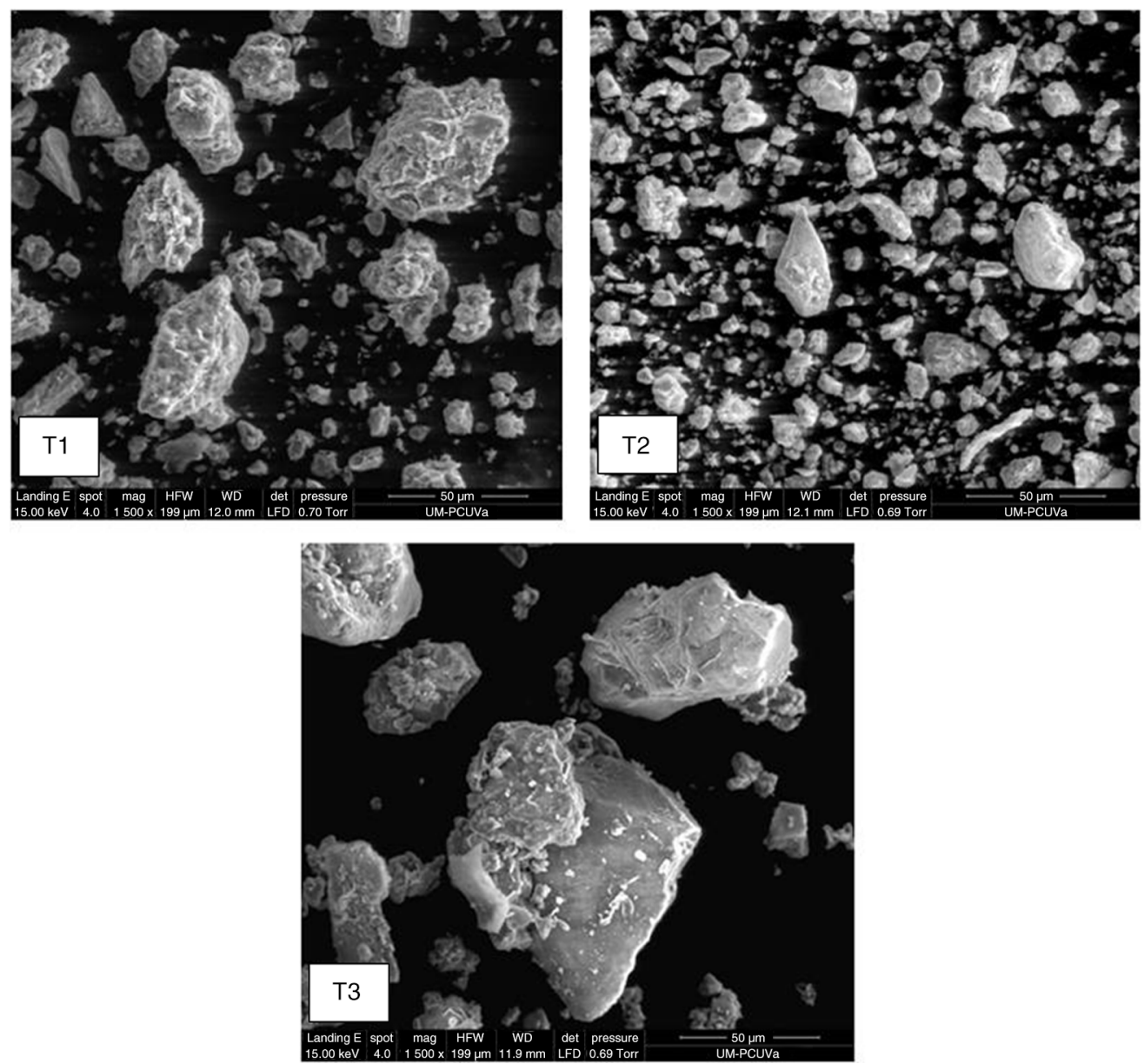

Fig. 5.-Microfotografías de los sedimentos de fondo en las tres etapas de análisis granulométrico. Sin tratamiento previo (T1), sin MO (T2), sin MO y con dispersante (T3).

\section{Conclusiones}

Tanto la variación de la abundancia de materia orgánica presente en los sedimentos de fondo del río Negro, así como de la conductividad eléctrica del agua, se encuentran reguladas principalmente por la estacionalidad climática regional. Se registraron aumentos marcados tanto del contenido de materia orgánica, como de concentración salina durante los períodos de aguas bajas. La concentración de sólidos en suspensión mostró un comportamiento inverso al de la conductividad, con disminución de la frecuencia en los períodos de aguas bajas.

Las técnicas experimentales empleadas en las determinaciones granulométricas de los sedimentos de fondo, indicaron un aumento de las fracciones más finas cuando se aplicaron técnicas dispersantes, confirmando la presencia de agregados sedimentarios en el medio natural. Se comprobó un aumento de las fracciones limo y arcilla al eliminar materia orgánica, y de la fracción arcilla al eliminar el efecto electroquímico producto de las altas concentraciones salinas. Las microfotografías tomadas en microscopio electrónico de barrido de los sedimentos de fondo en las tres etapas de análisis granulométrico, así como la relación entre la conductividad eléctrica del agua y la concentración de los sólidos suspendidos, permitieron corroborar lo observado en los análisis de tamaño de grano.

Por ello se asume que las determinaciones granulométricas de los sedimentos naturales deberían llevarse a cabo atendiendo a los objetivos de los ensayos y evaluando específicamente la conveniencia de tratamientos dispersivos. Para análisis ambientales 
vinculados al transporte de sedimentos, dicha práctica resulta desaconsejable al brindar una percepción de la dinámica sedimentaria diferente de la que se lleva a cabo en condiciones naturales del medio.

\section{AGRADECIMIENTOS}

Esta contribución se llevó a cabo mediante el apoyo económico del Proyecto PIP CONICET 11220100100406 (Consejo Nacional de Investigaciones Científicas y Técnicas).

\section{Referencias}

Ackermann, F. \& Schubert, B. (2007). Trace Metals as Indicators for the Dynamics of (Suspended) Particulate Matter in the Tidal Reach of the River Elbe. In: Sediment Dynamics and Pollutant Mobility in Rivers: an Interdisciplinary Approach (Westrich, B. \& Forstner, U., Eds.), Springer, Berlin, 296-304.

Berlamont, J.; Ockenden, M.; Toorman, E. \& Winterwero, J. (1993). The characterization of cohesive sediments properties. Coastal Engineering, 21: 105-128. http:// dx.doi.org/10.1016/0378-3839(93)90047-C

Carver, R.E. (1971). Procedures in sedimentary petrology. Wiley-Interscience. 653 pp.

Chetelat, B.; Liu, C.Q.; Wang, Q. \& Zhang, G. (2013). Assessing the influence of lithology on weathering indices of Changjiang river sediments. Chemical Geology, 359: 108-115. http://dx.doi.org/10.1016/j. chemgeo.2013.09.018

Clemente, J.M. \& Arocena, R. (2003). Zoobentos de diversos microhábitats en tres bañados de los Humedales del Este (Uruguay). In: Humedales de Iberoamérica (Neiff, J.J., Ed.), Programa Iberoamericano de Ciencia y Tecnología para el Desarrollo, 303-310.

Davide, V.; Pardos, M.; Diserensa, J.; Ugazio, G.; Thomas, R. \& Dominik, J. (2003). Characterisation of bed sediments and suspension of the river Po (Italy) during normal and high flow conditions. Water Research, 37: 2847-2864. http.//dx.doi.org/10.1016/S0043-1354 (03)00133-7

Fertonani, M. \& Prendes, H. (1983). Hidrología en áreas de llanura: aspectos conceptuales, teóricos y metodológicos, In: Hidrología de Grandes Llanuras, Vol. I. Argentina, UNESCO, 119-156.

Friese, K.; Schwartz, R. \& Krüger, F. (2007). Transport and Storage of River Sediment and Associated Trace Metals into Floodplains of the Elbe. In: Sediment Dynamics and Pollutant Mobility in Rivers: an Interdisciplinary Approach (Westrich, B. \& Forstner, U., Eds.), Springer, Berlin, 287-296.

Gibbs, R.J. (1983). Coagulation rates of clay minerals and natural sediments. Journal of sedimentary petrology, 53 (4): 1193-1203. http://dx.doi.org/10.1306/ 212F8341-2B24-11D7-8648000102C1865D
Habibi, M. (1994). Sediment transport estimation methods in river systems. PhD Thesis, University of Wollongong, $353 \mathrm{pp}$.

Hayter, E.J.; Bergs, M.A.; Gu, R.; McCutcheon, S.C.; Smith, S.J. \& Whiteley, H.J. (1999). HSCTM-2D, A Finite Element Model for Depth-Averaged Hydrodynamics, Sediment and Contaminant Transport. Report, National Exposure Research Laboratory, Office of Research and Development, U.S. EPA, Athens, Georgia. 220 pp.

Hostache, R.; Hissler, C.; Matgen, P.; Guignard, C. \& Bates, P. (2013). Modelling suspended-sediment propagation and related heavy metal contamination in floodplains: a parameter sensitivity analysis. Hydrology and Earth System Sciences, 18: 3539-3551. http://dx.doi.org/ 10.5194/hess-18-3539-2014

Huang, J.; Hilldale, R.C. \& Greimann, B.P. (2006). Cohesive Sediment Transport. In: Erosion and sedimentation manual. US Department of the Interior, Bureau of Reclamation, Denver, 1-46.

Lafleur. A.E.; Marea Llanos, M. \& Santa Cruz, J.N. (1980). Métodos granulométricos y determinaciones texturales. Normas y procedimientos. Instituto Nacional de Ciencia y Tecnología Hídricas (Buenos Aires), 9-20.

Iriondo, M.; Kröhling, D. \& Orfeo. O. (1998). Tropical Realm (provinces of Corrientes and Misiones). International Joint Field Meeting "Loess in Argentina: Temperate and Tropical". INQUA. Excursion Guide $\mathrm{N}^{\circ} 4,27$ pp.

Ledesma, L. \& Zurita, J.J. (1995). Los suelos de la Provincia del Chaco: Argentina, INTA (EERA Saenz Peña) Gobierno de la Provincia del Chaco. Report: 164 pp.

McLaren, P. \& Bowles, D. (1984). The effects of sediment transport on grain-size distributions. Journal of Sedimentary Petrology, 5: 457-470. http://dx.doi. org/10.1306/212F86FC-2B24-11D7-8648000102C1865D

McManu, J. (1995). Grain size determination an interpretation. In: Techniques in Sedimentology (Tucker, M. Ed.), Blackwell Science, 63-85.

Mehta, A.J.; Hayter, E.J.; Parker, W.R.; Krone, R.B. \& Teeter, A.M. (1989). Cohesive Sediment Transport. I: Process Description. Journal of Hydraulic Engineering, 115: 1076-1093. http://dx.doi.org/10.1061/ (ASCE)0733-9429(1989)115:8(1076)

Neiff, J.J. \& Orfeo, O. (2003). Aporte de materia orgánica de los humedales a ríos de sabana subtropical del Chaco, Argentina. In: Humedales de Iberoamérica (Neiff, J.J. Ed.), Programa Iberoamericano de Ciencia y Tecnología para el Desarrollo, 142-152.

OEA (Organización de los Estados Americanos). (1975). Estudio de la Cuenca Inferior del Río Bermejo. OEA-Gobierno Argentino, Tomo III, Recursos de la Tierra, $570 \mathrm{pp}$.

Orfeo, O. (1998). Temperate and tropical loess in Argentina: a textural comparison. International Joint Field Meeting. Loess in Argentina: Temperate and Tropical. INQUA. Abstracts: 21-22. 
Orfeo, O. (1999). Sedimentological characteristics of small rivers with loessic headwaters in the Chaco, South America. Quaternary International, 62: 69-74. http://dx.doi.org/10.1016/S1040-6182(99)00024-5

Patiño, C. \& Orfeo, O. (1997). Cambios en la calidad del agua de los ríos Negro y Salado (Provincia del Chaco, Argentina), en relación con su régimen hidrológico y salinidad. Comunicaciones de la Secretaría General de Ciencia y Técnica.

Poi de Neiff, A.; Patiño, C.; Neiff, J.J. \& Ramos, A.O. (2003). Calidad del agua en el tramo bajo del río Negro (Chaco, Argentina). FACENA, 19: 67-85.

RAMSAR. Humedales Chaco. Ramsar Sites Information Service database. (último acceso 22/10/2014). Disponible en: https://rsis.ramsar.org/ris/1366

Ruberto, A.R. (1999). Hidroquímica de la cuenca del río Negro (Chaco). Tesis de Maestrando. Universidad Nacional del Nordeste (Argentina). 37 pp.
Smith, G.D. (1986). The Guy Smith Interviews: Rationale for concepts in Soil Taxonomy. SMSS Tech. Mon., 11.

Spalletti, L.A. (1986). Nociones sobre transporte y depositación de sedimentos clásticos. Revista de la Facultad de Ciencias Naturales y Museo, Universidad Nacional de la Plata, Serie Técnica y Didáctica, 13. $102 \mathrm{pp}$.

Suárez, P.; Sartirana, M. \& Orfeo, O. (2010). Caracteres sedimentológicos de ambientes fluviales de la llanura subtropical chaqueña (Argentina). Acta Geológica Lilloana, 22: 34-45.

Symader, W.; Bierl, R.; Kurtenbach, A. \& Krein, A. (2007). The relevance of River Botton Sediments for the transport of Cohesive Particles and Attached contaminants. In: Sediment Dynamics and Pollutant Mobility in Rivers: an Interdisciplinary Approach (Westrich, B. \& Forstner, U., Eds.), Springer, Berlin, 269-279. 